\title{
Digitally Developing Medieval Fortifications
}

\author{
Andreas Georgopoulos a , Margarita Skamantzari ${ }^{\text {b }}$, Sevi Tapinaki ${ }^{\text {c }}$ \\ Laboratory of Photogrammetry - National Technical University of Athens, Athens, Greece \\ adrag@central.ntua.gr; ${ }^{\mathrm{a}}$ mskamantz@ central.ntua.gr; ${ }^{\mathrm{c}}$ sevi@ survey.ntua.gr
}

\begin{abstract}
Modern technological advances have enabled digital automated methods to be applied for accurate and detailed documentation. Such techniques include image based modelling and terrestrial laser scanning. They can easily be adapted to perfectly suit the documentation needs for small and large objects. In this paper the digital geometric documentation of two medieval fortifications using such contemporary methods is briefly described. These are the western part of the Castle of Chios and an important part of the medieval Rhodes fortifications. The purpose of the geometric documentation for both cases was the restoration of these parts, hence detailed documentation was necessary. Conventional two-dimensional plans with digital orthophotographs were produced and from them conventional line drawings were required for the case of Chios castle. Both castle parts included cylindrical bastions, whose projection on two dimensional plans is obviously deformed. Such non-planar parts constitute a special challenge for the geometric documentation. To solve this problem the three-dimensional digital documentations of these parts were developed by using suitable development routines. Thorough presentation of all considerations for these developments are presented and the implementations are briefly described. The results of these developments are evaluated for their usefulness, accuracy, and efficiency as digital documentation products.
\end{abstract}

Keywords: Geometric documentation, image-based modelling, terrestrial laser scanning, digital development.

\section{Introduction}

All over Greece and especially along its mainland and island coastline, one can find hundreds of defensive fortifications and structures. A large number of them have been built since the ancient and Roman times, but their construction was boosted during the Byzantine and, most of all, the Genovese era. A number of these castles was modified by the Ottomans to suit the warfare of the time. These fortifications include castles, towers, fortified monasteries, and watch towers. Today these monuments are under the responsibility of the Greek Ministry of Culture. These numerous fortifications are subject to decay mainly due to natural hazards A large part of them is facing the sea, thus presenting severe structural and restoration problems. Earthquakes and strong winds, especially on mountain tops and at sea fronts, are a constant source of destruction. For that reason, these structures should be thoroughly protected, restored and, most importantly, geometrically documented, as the Venice Charter dictates (ICOMOS, 1964). About ten years ago the Ministry of Culture devoted funds for the documentation and restoration of 36 such seaward situated on islands and 
ports in Greece under a project called "Kastron Periplous", i.e. Castles' Circumnavigation.

Nowadays, geometric documentation of cultural heritage may be performed with far more efficient technological means than even a decade ago. Digital imagery, terrestrial laser scanning and automated photogrammetric and computer vision algorithms have enabled experts to produce stunning 2D and 3D documentation products, such as orthophotos and 3D models. These alternative products, however, have not yet been widely accepted by the conservation and restoration community. Two-dimensional line and vector drawings are still required, and will still be, for the restoration actions. This is by no means negative, as the information content in the contemporary digital products is huge and, sometimes, too difficult to extract in such a form as to be exploited for restoration actions.

Geometric documentation is the process of acquiring, processing and presenting information, which determines the form, size and position of a cultural heritage asset in 3D space and at a given moment in time. In this paper the geometric documentation of specific parts of two fortifications in Greece is briefly presented and special focus is given on the geometric development of curved surfaces, i.e. cylindrical bastions, as their simple orthogonal projections on a $2 \mathrm{D}$ vertical plane is inevitably deformed and, consequently, useless for restoration purposes.

The structure of the paper is as follows: In section 2 the two fortifications are briefly described. In section 3 geometric documentation methodology is presented, while in section 4 our approach for developing the surfaces is explained. In section 5 the results are presented and evaluated and finally conclusions are drawn in section 6 .

\section{Case Studies}

In this paper the digital geometric documentation of two such fortifications using state of the art techniques is briefly described. These are (a) the western part of the Castle of Chios and (b) an important part of the medieval Rhodes fortifications (Fig. 1).

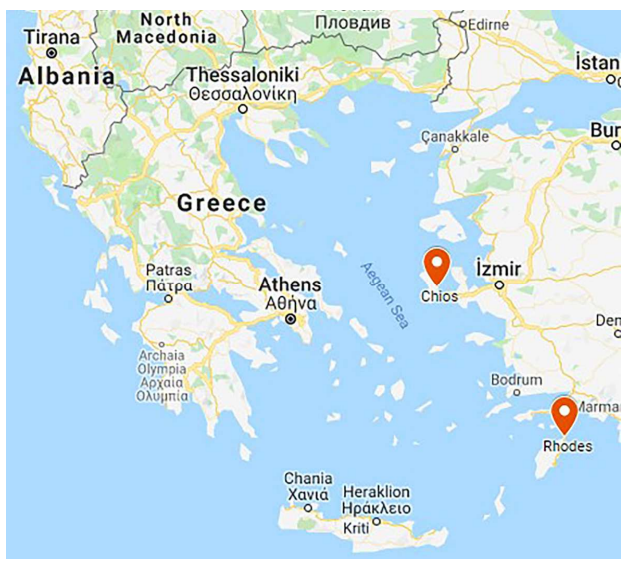

Fig. 1. Location of Chios and Rhodes (Google Maps).

The purpose of the 3D geometric documentation for both cases was the restoration of these parts, hence detailed documentation was necessary. Conventional two-dimensional plans with digital orthophotographs were specified and delivered based on the production of the three-dimensional models of the structures and consequently from them conventional line drawings were required for the case of Chios castle (Tryfona, et al., 2016). Both castle parts included cylindrical bastions, whose projection on two dimensional plans is obviously deformed. Such non-planar parts form a difficulty for the geometric documentation.

\subsection{The Castle of Chios}

Chios is an island in the Eastern part of the Aegean Sea and has a long history especially during the medieval period when Genovese conquerors occupied almost all islands in the Aegean Sea.

The fortress (Fig. 2) covers an area of 180000 $\mathrm{m}^{2}$ and today its walls encircle a residential complex of 650 inhabitants. The inhabitation of the castle area is witnessed at least since the Hellenistic times, i.e. fourth century BC. According to the excavations, the occupation and use of the fortress continued during the Roman and preByzantine times. According to historic references the present castle of Chios is identical to the well-known fortress that the Genovese ruler of Chios, Martino Zaccaria, started to build in 
1328. Today the form of the fortress of the Chios Castle is the result of constant renovations, additions, and enhancements during the long existence of the monument. Its walls both in land and at sea form an irregular pentagon with strong bastions, eight of which are still preserved (Fig. 2) (http://odysseus.culture.gr).

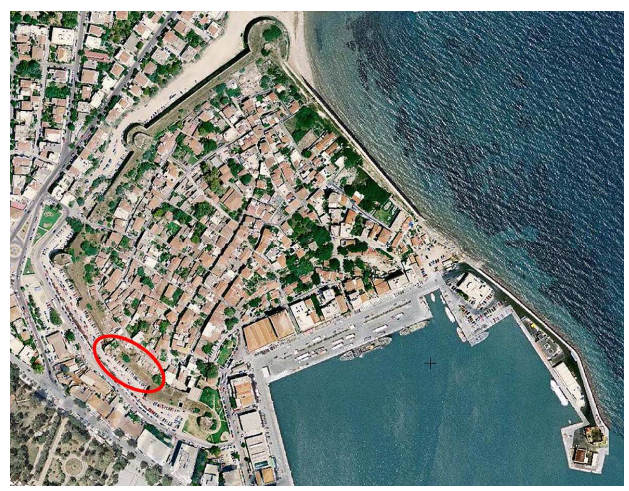

Fig. 2. Aerial view of The Fortress and Port of Chios (Google Earth).

The object of the present study was a large linear part of the walls at the southwestern side together with the cylindrical bastion with a square tower on top (Figs. 2 and 3).

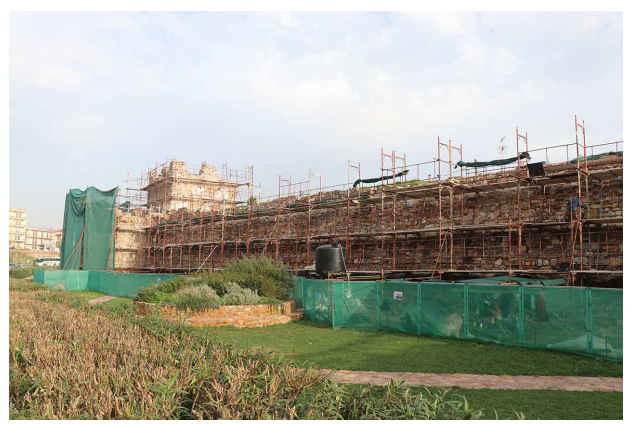

Fig. 3. Part of the Castle of Chios.

\subsection{The Gate of the Cannons in Rhodes}

The Gate of the cannons is located at the southeast corner of the Grand Master's Palace and provided access to the battle positions on the ramparts of the walls surrounding and fortifying the Medieval City. It was constructed during Jean de Lactic's Magistery (1437-1454) and bears his Coat of Arms (Fig. 4).

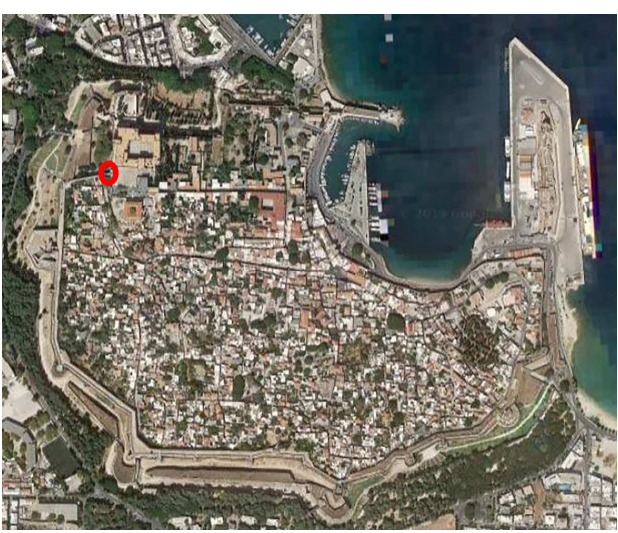

Fig. 4. Aerial view of the Castle of Rhodes (Google Earth).

The Gate was symmetrically protected by two round cylindrical towers, one on each side, a fact that indicates the presence of a drawbridge connecting the Gate with the ramparts over the moat (Fig. 5).

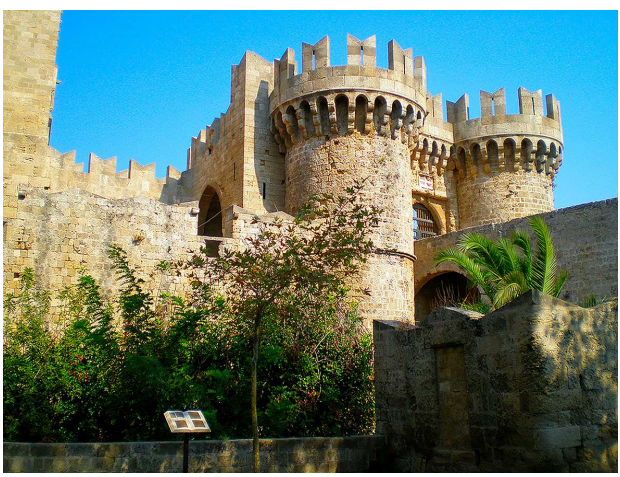

Fig. 5. The Cannon Gate in Rhodes (http://rodosisland.gr).

Crossing the Gate, the garrison of the Palace could have immediate access to the ramparts and the bastion, which faces the famous Gate d' Amboise, to defend the Palace. The bastions were equipped with cannons, hence the name of the Gate However, many historians have stated that those cannons were added much later as a decorative element (http://www.medievaltown. gr/item/gate-of-the-canons/). 


\section{Methodology for the Geometric Documen- tation}

The geometric documentation of such complex fortified constructions presents challenges and difficulties, especially when it comes to the development of curved, cylindrical, conical, spherical, parabolic or ellipsoidal surfaces. The typology and constant change and evolution of fortifications through the ages give significant information about their structure, their component elements, the function, the form, shape and use of them through the ages. As a result, all these elements should be taken into consideration when it comes to documentation in general, since except from the user's needs, the typology of a monument and its architectural details play a fundamental role to the decisions and choices of the documentation and can even dictate the final products. Throughout the years many examples and applications can be found in the literature concerning the documentation of fortifications and the creation of both vector and raster products as well as developments derived from photogrammetric data. According to Tapinaki et al 2019, raster and vector products for documentation of fortification monuments can be used in a variety of applications; in studies of archaeological or architectural interest (Drap, et al., 2005; Ringle, et al., 2005; Gianniou, et al., 2007; Chiabrando, et al., 2009; Vrettou, Georgopoulos, 2016), for designing maintenance and restoration work (Almagro Gorbea, Orihuela Uzal, 2015; Kersten, et al., 2015), structural analysis of constructions, digital reconstruction of damaged or destroyed monuments (Grussenmeyer, Yasmine, 2003; RodríguezNavarro, Verdiani, 2013) for historical interpretation, and for upgrading the touristic product of a region.

Nowadays modern technological advances have enabled digital automated methods to be applied for accurate and detailed 3D documentation of archaeological sites and monuments (Georgopoulos, Ioannidis, 2004). Meanwhile, different strategies, techniques and methods can be applied, and various software can be used for each case according to the final products, the required accuracy, the available equipment, means and, of course, budget.
For the 3D geometric documentation of both the Castle of Chios and the Cannon Gate in Rhodes the same general methodology and workflow was followed, including close-range automated photogrammetry, image-based modelling, terrestrial laser scanning and topographic surveys. The combination of these methods has been proven the ideal solution for cases like these in order to achieve complete and accurate results. In particular, the equipment that was used included a standard total station (Topcon GPT3003N), a time-of-flight pulse-based 3D Terrestrial Laser Scanner (Leica Scanstation 10), a time-of-flight phase shift 3D Terrestrial Laser Scanner (FARO Focus 110) two DSLR cameras (Canon EOS 6D and Canon 80D with $8-15 \mathrm{~mm}, 24 \mathrm{~mm}$ and $18-55$ $\mathrm{mm}$ lenses) and an unmanned aerial vehicle (DJI Phantom 4 Pro).

Firstly, a trigonometric network was established at each fortified structure in order to establish an arbitrary local coordinate reference system. The networks were adjusted, and the station coordinates were determined with an accuracy of a 2-3 $\mathrm{mm}$. All measurements and data acquired were referenced to the same system in order to avoid deviations of the shape or size of the monument. Moreover, all ground control points (GCPs), laser scanner targets and the additional, necessary points were measured and referenced to these local systems.

To produce the 3D models image-based modelling was employed. Suitable digital images, both aerial and terrestrial, were taken from key positions and directions according to the part of each fortified structure that should be documented and the difficulties that each object presented in terms of shape, obstacles, illumination etc. The UAV was used in both cases to document the upper parts of the fortifications and the parts that were difficult or unattainable to get digital images with the handheld DSLR cameras. Images were taken in such a way to fulfil the Structurefrom-Motion (SfM) requirements, i.e. image every detail on at least two adjacent images.

Finally, the walls and the bastion of the Castle of Chios as well as the two cylindrical towers of the Cannon Gate of Rhodes were scanned from multiple scanner positions in order to overcome 
any eventual lack of information that could arise and record all the necessary details of the monuments.

The data processing process concerned mostly the digital images, which were processed with the Image Based Modelling (IBM) software Agisoft Photoscan v.1.4.2. to produce the detailed 3D textured model as well as the orthophotos for each case study.

In each case, the images were loaded, examined, evaluated and masks were applied on those that it was considered necessary to exclude the obstacles and unnecessary noise for the rest of the processing. The next step of the process was the automatic detection of the pre-marked targets to facilitate the alignment, i.e. the orientation of the images and of course the scaling and georeferencing of the point cloud using the determined GCPs. Any deviations that arose at this point had to be checked and eliminated. The scale of the products dictates the final accuracy which in the case of Chios was $6,25 \mathrm{~mm}$ (scale 1:25) while in the case of Rhodes was $12,5 \mathrm{~mm}$ (scale 1:50).

Then the dense point cloud was generated by the IBM software and further processed with Geomagic Wrap 2017. The clean and filtered dense point cloud from Photoscan was combined with the registered scanned point clouds to produce a complete surface description. The final point cloud generated for each monument was then converted to an accurate, detailed and smooth 3D polygon surface. Each mesh was then imported once again into the IBM software to build the textured model as well as the orthophotos. Subsequently, the 2D vector drawings were produced by suitably tracing the orthophotos within a CAD environment. For the case of the medieval fortress of Chios the following orthophotos, 2D and architectural vector drawings were produced:

1) The bird's eye view of the monument.

2) The development of the bastion.

3) The four façades of the tower.

4) The façade of the walls.

5) One vertical section intersecting both the bastion and the tower in the middle.
6) One vertical section intersecting the walls in the middle.

For the case of the Cannon Gate in Rhodes the following products were needed and produced:

1) The bird's eye view of the monument.

2) The development of the west façade.

3) The four façades of the monument.

In both these cases there was the need for the development of the cylindrical surfaces, specifically the bastion of Chios and the two towers of the Cannon Gate. Nowadays, there is a variety of software that can produce surface developments. It was necessary to decide which method should be used for the process in a short time, providing a complete, accurate and detailed result in orthophoto. According to previous case studies and after thorough research Agisoft Photoscan v.1.4. was chosen among 3DReshaper, Cloud Compare and Geomagic Wrap 2017, for the developments and the required orthophotos as it is described below.

\section{Digital Developments}

For the development of the bastion of the Castle of Chios the generation of sections at different levels of the bastion led to the conclusion that its surface is not entirely cylindrical but slightly conical.

The deviation from the perfect cylinder occurs in the upper part of the bastion where the angle of inclination from the vertical was determined at 0,0006 degrees in the top $1,5-2,0 \mathrm{~m}$ out of the $10 \mathrm{~m}$ in total. Thorough geometric investigation led to the conclusion that if this deviation were neglected, it would result to insignificant distortion to the projected segment lengths for the required scales (1:40 and 1:25). Therefore, it was decided that the bastion's surface could be considered as a cylinder.

The development of the bastion's surface also included two planar parts at the points where the cylindrical surface met the planar surfaces of the fortification walls. In Fig. 6 the horizontal section is shown, and the bold numbers indicate where the surface changes from planar to cylindrical. 


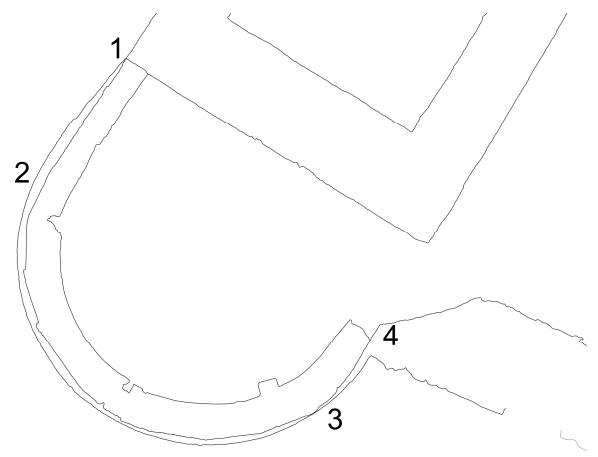

Fig. 6. Horizontal Section of the bastion in Chios.

The development of the whole bastion is shown in Fig. 7.

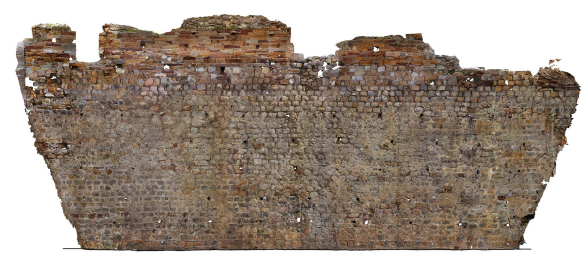

Fig. 1. The development of the cylindrical bastion of the Castle of Chios.

The development of the Cannon Gate towers was more challenging, because of the different and complicated shapes composing them. The eastern façade is planar, so only the production of a simple orthophoto was required (Fig. 8).

The western façade, on the other hand, consists of three parts, the Gate in the centre and two almost symmetrical towers on either side. Each part is composed by the wall at its lower part, the protruding battlements on top and the corbels supporting them (Fig. 5).

On each of the three parts, both the surface of the wall and that of the battlements have the same shape. The middle part of the main Gate is planar, while the two towers are cylindrical. The corbels, on the other hand, present particularly complicated shape. Each corbel is always perpendicular to the surface of the wall, either planar or cylindrical. Consequently, each group of corbels follows the shape of the wall and battlements (Fig. 5). As a result, their front surface does not belong to a unique surface able to be easily developed. Hence, different approaches were necessary for each part.

A thorough geometric analysis and examination of the whole object was performed with several horizontal and vertical sections of the 3D model. This investigation led to the conclusion that the towers are not symmetrical. For the northern tower both the wall and the battlements are cylindrical while the southern one consists of cylindrical and planar surfaces. In Fig. 8 these horizontal sections are shown, and the bold numbers indicate where the surface changes from planar to cylindrical.

Standard orthophotos were produced for the planar parts, while the cylindrical parts were developed using the specific methodology described above.

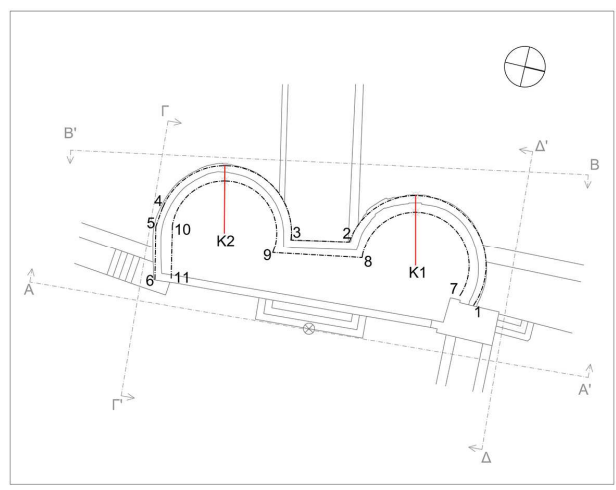

Fig. 8. Horizontal Sections of Cannon Gate in Rhodes.

The arched corbels are protruding from the wall and are composed of three parts of the same shape but with different dimensions. The smaller one is the lower part and starts from the wall while the biggest one is the upper part, reaching the face of the battlement (Fig. 9). Therefore, the corbel consists of three surfaces, two planar ones and perpendicular to the wall and the triple curved front surface. The development of such a surface would lead to a very complex and incomprehensible drawing.

Due to the complicated shape of each corbel and after discussion with the supervising architects, it was decided to deal only with the façade of each one, ignoring the surfaces perpendicular to the wall, which in any case would not be entirely 
visible. The main reason was that the final product would be very cluttered with unnecessary information.

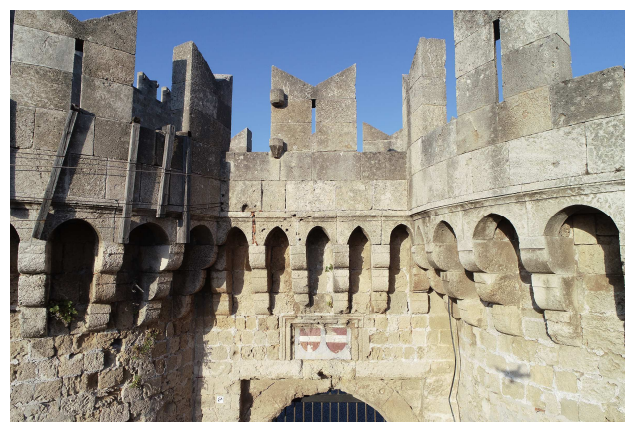

Fig. 9. The corbels.

Finally, the suitable vertical plane for each corbel was determined and the orthophoto of each one was produced separately.

The development of the whole object is shown in Fig. 10, where the three parts are positioned at a distance, so that the drawing becomes more legible. Furthermore, the wall between the corbels has no raster for the same above explained reasons. The numbers correspond to the ones of Fig. 8.

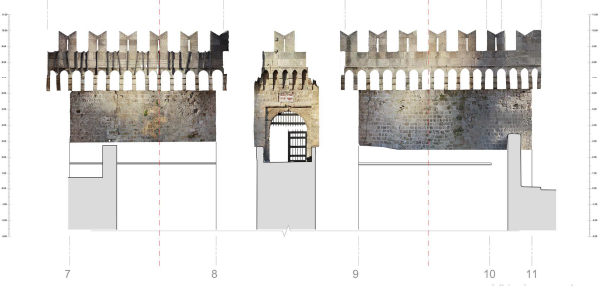

Fig. 10. The development of the Cannon Gate in Rhodes.

\section{Evaluation of results}

The criteria used for the quality control of the products, i.e. the 3D model, the orthophotos and the developments, are those of conformity, completeness, and accuracy.

The concept of conformity is the degree of faithful depiction of the object, while the concept of completeness, the degree of coverage of the object documented. The completeness and con- formity check are usually performed by visual observation which entails a high degree of subjectivity. However, in both cases the developments satisfied the restoration experts.

The spatial accuracy of the 3D models, from which the developments were produced, was assessed by examining the reprojection errors of the Ground Control Points (GCPs). Regarding the accuracy of the model, the average reprojection error of the GCPs' coordinates in the model in the case of the Chios castle is $\sigma_{x y z}= \pm 7,5 \mathrm{~mm}$, which is considered acceptable. In the case of the Cannon Gate in Rhodes this error was $\sigma_{\mathrm{xyz}}=$ $\pm 6,3 \mathrm{~mm}$.

The accuracy of the orthophotos was assessed by examining the a posteriori standard error between the geodetic coordinates as measured in the field and their corresponding values in the produced orthophoto of the GCPs used as check points. The a posteriori error was $\sigma_{\mathrm{x}}= \pm 5,7 \mathrm{~cm}$ and $\sigma_{y}= \pm 3,3 \mathrm{~cm}$. Since the GCPs were more or less uniformly distributed, the errors may be considered that express the accuracy of the points forming the entire model.

\section{Conclusions}

It has been clearly demonstrated that using contemporary digital means of documenting Cultural Heritage objects and in this case fortifications, in particular, it is possible to easily produce alternative drawings, useful for the conservation and restoration studies. Developments of complicated surfaces present the merit of directly extracting metric information as they represent the objects in orthogonal projection without deformations.

Investigations are already underway on how non developable surfaces could also be developed using contemporary means. This will be achieved by employing cartographic mathematical projections. In this case the non-developable surface elements are projected onto a suitably determined developable surface (plane, cylinder, or cone) and subsequently this surface is developed. This process involves inevitable controlled deformations which are known beforehand, as it is the case with maps. 


\section{Bibliography}

Almagro Gorbea, A.; Orihuela Uzal, A. (2015). "Métodos para documentar y restaurar zonas de difícil acceso en el Castillo de Salobreña (Granada)", in Defensive Architecture of the Mediterranean XV to XVIII centuries, vol. 1, pp. 287-290.

Chiabrando, F.; Bernardi, M.L. De.; Curetti, S. (2009). "Integration of low-cost geomatics techniques to support the architectural project the Perlo castle area survey”, in XXII CIPA Symposium Kyoto, Japan.

Drap, P.; Durand, A.; Seinturier, J.; Vannini, G.; Nucciotti, M. (2005). "Full XML documentation from photogrammetric survey to 3D visualization. The case study of Shawbak castle in Jordan", in XX CIPA International Symposium Torino, Italy.

Georgopoulos, A.; Ioannidis, Ch. (2004). "Photogrammetric and Surveying Methods for the Geometric Recording of Archaeological Monuments", in FIG International Week, Athens, in http://www.fig.net/pub/athens/papers/wsa1/WSA1_1_Georgopoulos_Ioannidis.pdf.

Gianniou, P.; Georgopoulos, A.; Tsakiri, M.; Della, K. (2007). "The documentation of the medieval entrance of the Rhodes fortification complex", in XXI International CIPA Symposium, Athens Greece.

Grussenmeyer, P.; Yasmine, J. (2003). "The restoration of Beaufort castle (South Lebanon) A 3D restitution according to historical documentation", in XIX International CIPA Symposium, Antalya Turkey.

ICOMOS. (1964). Venice Charter, in https://www.icomos.org/charters/venice_e.pdf.

Kersten, T.; Mechelke, K.; Maziull, L. (2015). „3D model of Al Zubarah fortress in Qatar - Terrestial Laser Scanning vs Dense Image Matching", The International Archives of the Photogrammetry, Remote Sensing and Spatial Information Sciences, vol. XL-5/W4, pp. 1-8.

Rodriguez-Navarro, P.; Verdiani, G. (2013). "Digital survey and interpretation of a fortification fragment: the Cadi bridge at the feet of the Alhambra hill, Granada", in Digital Heritage International Congress (DigitalHeritage), vol. 2, pp. 363-366.

Ringle, K.; Nutto, M.; Teschauer, O.; Mohn, C. (2005). "Integration of historical plans into a modern facility management system taking the castle of Heidelberg as an example", in XX CIPA International Symposium Torino, Italy.

Tapinaki, S.; Skamantzari, M.; Chliverou, R.; Evgenikou, V.; Konidi, A.M.; Ioannatou, E.; Mylonas, A.; Georgopoulos, A. (2019). "3D Image Based Geometric Documentation of a Medieval Fortress", Int. Arch. Photogramm. Remote Sens. Spatial Inf. Sci., XLII-2/W9, pp. 699-705, in https://doi.org/10.5194/isprs-archives-XLII-2-W9699-2019.

Tryfona, M.S.; Georgopoulos, A. (2016). "3D Image Based Geometric Documentation of the Tower of Winds", Int. Arch. Photogramm. Remote Sens. Spatial Inf. Sci., XLI-B5, pp. 969-975.

Vrettou, F.; Georgopoulos, A., (2016). "Castle Penteskoufi: Geometric Documentation. Proceedings of the International Conference on Modern Age Fortifications of the Mediterranean Coast", in FORTMED 2016. Defensive architecture of the Mediterranean XV to XVIII centuries, Vol. IV, pp. 317-323. 